\section{CAMPESINADO Y GESTIÓN PLURIACTIVA DEL ECOSISTEMA: UN MARCO TEÓRICO PARA EL ANÁLISIS DEL III Y II MILENIOS A.C. EN LA MESETA PENINSULAR}

\author{
PEASANTRY AND MULTIACTIVE \\ ECOSYSTEM MANAGEMENT: A \\ THEORETICAL FRAMEWORK FOR THE \\ ANALYSIS OF THE IBERIAN MESETA \\ DURING THE III AND II \\ MILLENNIA B.C.
}

\section{PEDRO DÍAZ-DEL-RÍO ESPAÑOL $\left({ }^{*}\right)$}

\section{RESUMEN}

Este trabajo propone un marco teórico alternativo para el análisis de la Prehistoria reciente de la Meseta peninsular, incidiendo en el caracter pluriactivo de la racionalidad económica campesina. Desde esta perspectiva, se discuten diversas hipótesis en las que prevalece el elemento pastoril como factor determinante en la economía prehistórica. Como conclusión, se presenta la evolución social de la Meseta como una anomalía histórica, en la que un tipo específico de gestión económica mitigó el desarrollo de desigualdades sociales permanentes.

\begin{abstract}
The present paper discusses the Meseta's Late Prehistory from an alternative theoretical framework, focussing on the multiactive character of peasant economic rationality. From this point of view, recurrent interpretation of the dominance of pastoralist in prehistoric conomies are discussed. In conclusion, the social evolution of this area is seen as an historical anomaly, in which a specific form of ecosystem management restrains the development of permanent social inequalities.
\end{abstract}

(*) Cl Conde de la Cimera 4, 3-3. 28040 Madrid. El artículo fue remitido en su versión final el 14-XI-95.
Palabras clave: Calcolítico. Edad del Bronce. Campesinos. Pastores. Historia. Desigualdad social.

Key words: Chalcolithic. Bronze Age. Peasants. Pastoralism. History. Social inequalities.

\section{OBJETIVO DEL TRABAJO}

El presente trabajo pretende defender la viabilidad del uso del concepto de «campesinado" para el análisis de las sociedades de la Prehistoria reciente en la Península Ibérica. Su objetivo final es romper con el antagonismo pastor-agricultor presupuesto por la gran mayoría de los prehistoriadores a la hora de interpretar las sociedades del III y II milenio a.C. Esta tentativa no es en forma alguna novedosa, encontrando antecedentes directos en trabajos como el de Chapman (1979) sobre el megalitismo peninsular o de Lewthwaite (1981) sobre la trashumancia mediterránea. Sin embargo difiere en el enfoque utilizado, reivindicando la potencialidad analítica y descriptiva de las herramientas desarrolladas a lo largo de más de dos décadas por la corriente multidisciplinar denominada «Estudios Campesinos». 
Para confrontar el modelo propuesto. se han seleccionado dos interpretaciones actualmente vigentes. La primera postula la existencia de una economía fundamentalmente ganadera para las poblaciones del III y II milenio a.C., utilizando para ello una perspectiva normativista. La segunda, defendida desde una perspectiva procesual por R.J. Harrison, postula la existencia de un modo pastoril integrado durante la Edad del Bronce en la Meseta peninsular.

El marco geográfico, en el cual los tópicos en torno a las sociedades de carácter pastoril han sido reiteradamente utilizados, es la Meseta peninsular. Dada la amplitud del espacio propuesto, se recurre como puntual objeto de estudio al área de la Comunidad de Madrid, por cuanto, a pesar de sus especiales características, puede considerarse como paradigmática en la defensa normativista de una economía ganadera para la Prehistoria reciente de la Meseta.

\section{MADRID COMO CASO DE ESTUDIO: LAS BASES EMPÍRICAS DE UNA INTERPRETACIÓN NORMATIVISTA}

La actual Comunidad de Madrid es una división administrativa situada en el área central de la Península Ibérica. Queda delimitada al norte por el Sistema Central y al sur por el cauce del río Tajo, límites que establecen una de las principales características geográficas del área: el contraste entre la sierra y las fértiles cuencas sedimentarias de los afluentes del Tajo.

Las intervenciones arqueológicas desarrolladas en la región se remontan a finales del siglo XIX, siempre dependientes de las obras de crecimiento urbano y explotación industrial. Esta dependencia, vigente en algunos casos, ha creado un singular registro arqueológico, en gran medida deficiente dada la ausencia de excavaciones estratigráficas (Domínguez et alii, 1993). Asimismo, ha limitado las interpretaciones a áreas muy concretas, en especial a los cauces fluviales.

Estas y otras muchas deficiencias han mediatizado la interpretación normativista imperante, fundamentada en la inducción directa del registro arqueológico. Por una parte, la falta de una periodización basada en referentes estratigráficos ha llevado construir la misma sobre bases exclusivamente morfotipológicas. Por otra, la información arqueológica respecto a algunas cuencas sedimentarias ha permitido defender la hipótesis del carácter primordialmente pastoril de la economía durante la Prehistoria reciente.

Los indicadores arqueológicos utilizados para interpretar la sociedad del III y II milenio a.C. en Madrid son los siguientes:

- La eclosión de yacimientos en llano durante el Calcolítico y la continuidad de un patrón de asentamiento lineal a lo largo de toda la Edad del Bronce. El absoluto desconocimiento de los aspectos económicos de la neolitización en el interior de la Península Ibérica, y en particular en la región de Madrid (Antona, 1987), convierte al Calcolítico en la primera fase en que abunda el registro arqueológico. Los yacimientos se distribuyen a lo largo de los cauces fluviales, en algunos casos distanciados entre sí no más de un kilómetro (p.e. Blasco, 1994; Blasco et alii, 1991: Almagro y Benito-López, 1993).

- La continuidad de asentamientos puntuales en altura sin un patente carácter defensivo. Aunque tradicionalmente se han considerado una característica relevante del Bronce Final, los yacimientos en altura, generalmente en cerros testigo o antecerros, han sido documentados para el Calcolítico (Martínez Navarrete, 1984), Bronce Medio (1) y Bronce Final (Almagro y Fernández-Galiano, 1980).

- La aparición en ambos tipos de yacimientos de acumulaciones de subestructuras, conocidas como «fondos de cabaña». Generalmente, y salvo raras excepciones, se trata de amplias áreas en las que aparecen elementos interfaciales sin relación de superposición, rellenos de diversos tipos de depósitos. Son, por tanto, yacimientos no estratificados si por ello entendemos que no existen superposiciones físicas entre los diversos períodos cronológicos. Con escasísimas excepciones, como el yacimiento de El Capricho (Rus y Velasco, 1993), el Ventorro (Priego y Quero, 1992) o el Arenero de Soto (Pernia y Leira, 1992), los únicos restos de estructuras de habitación son puntuales hallazgos de agujeros de poste dispuestos de manera dispersa y sin relación estratigráfica. Este fenómeno ha sido tradicionalmente achacado a la existencia de los

(1) Aunque inéditas, las prospecciones llevadas a cabo dentro del programa de Carta Arqueológica de la Comunidad de Madrid han localizado puntuales yacimientos del Bronce Medio en altura. Vid. p.e. la Carta Arqueológica del Término Municipal de Villalbilla, memoria redactada por P. Diaz-delRío, L. Román y E. Serrano, depositada en la Dirección General de Patrimonio Cultural de la Comunidad de Madrid. 
nunca analizados procesos postdeposicionales. mientras se han obviado las evidentes deficiencias que acarrea la falta de aplicación de técnicas estratigráficas a la hora de extraer el registro arqueológico. A pesar de todo ello, el problema no es exclusivo de la región madrileña, ni siquiera de la Meseta, distribuyéndose a lo largo de la Península Ibérica en aquellos lugares donde no aparecen construcciones en piedra.

Sin embargo, este registro no conduce directamente a la formulación del caracter pastoril de los pobladores de la Meseta Peninsular. El origen de la interpretación puede remontarse a la explicación clásica del «fenómeno Campaniforme» y el posterior desarrollo de la «cultura de Cogotas I» durante el Bronce Final. El normativismo, al huir de cualquier tentativa evolucionista, recurre (consciente o inconscientemente) a la difusión y el contacto a la hora de explicar la aparición de algun tipo específico de resto arqueológico en diversos contextos peninsulares (p.e. cerámicas campaniformes o excisas de Cogotas I). Dentro de este clásico marco difusionista, la trasmisión cultural y la distribución geográfica de "fósiles directores» era consecuencia directa de la movilidad de las poblaciones, siendo el recurso más frecuente el uso del tópico etnográfico del pastor nómada o trashumante. Ya establecidos los fundamentos de la difusión de los fenómenos Campaniforme y Cogotas I, no resultó difícil su extrapolación al resto de los períodos que conforman la Prehistoria reciente de la Meseta Peninsular.

De esta forma, la corriente normativista ha tenido en cuenta ciertos aspectos del registro arqueológico madrileño, de entre los que destacan:

- La existencia de humedales en las terrazas fluviales, las cuales conservan actualmente pastos frescos a lo largo de todo el año. Esto permite suponer un movimiento de población basado en una economía principalmente de pastoreo (p.e. Blasco, 1993; 1994). Se utiliza como elementos de apoyo a dicha interpretación la distribución lineal del hábitat a lo largo de los cauces fluviales, la supremacía del ganado ovicaprino en los análisis faunísticos y las supuestas escasas condiciones de las vegas para el desarrollo de una actividad agrícola (p.e. Blasco et alii, 1994: 64).

- La inexistencia de construcciones en piedra como indicador de la escasa estabilidad del hábitat.
- La dualidad en la gestión económica durante la totalidad de la Prehistoria reciente. Frente a la actividad pastoril supuesta para los pobladores del llano, las interpretaciones normativistas inciden en el componente agrícola y comercial de los asentados en altura (p.e. Blasco, 1992). Implícitamente, la interpretación conlleva una dualidad de poblaciones.

Gran parte de estas explicaciones se fundamentan en diversos tópicos etnográficos, utilizados de-manera recurrente por los investigadores a la hora de defender una economía de pastoreo para la Prehistoria reciente en Europa, como son:

- La errónea dicotomía supuesta entre agricultura-sedentarismo y pastoreo-movilidad. La diferencia clave entre una sociedad agrícola o pastoril se encuentra, al margen de la posible movilidad del grupo, en el anclaje sobre el cual se sitúan las relaciones de producción dominantes: tierra o ganado (Ingold, 1987). La pronunciada sedentarización de la población rural no representa un rasgo diacrónico especificamente distintivo de ningún tipo de sociedad precapitalista, por lo que un patrón lineal de distribución del hábitat no puede ser utilizado como indicador determinante del carácter pastoril de la misma. La propia dinámica interpretativa lleva a casos extremos, como considerar la presencia de un registro arqueológico con más de dos períodos de ocupación la constatación de las «idas y venidas» de diversos grupos de pastores.

Otro referente empírico de este mismo tópico es el recurso a la ausencia de construcciones en piedra, lo cual implica considerar que un pastor deja menos huella arqueológica que un agricultor. Con ello se anula todo posible grado de determinación generado tanto por el acceso directo a materiales constructivos como por la tradición cultural preexistente.

- La supuesta contraposición entre ganadería y agricultura. Uno de los elementos fundamentales que tradicionalmente han unido a las poblaciones campesinas mediterráneas ha sido la constante vinculación del elemento ganadero a los factores estrictamente agrícolas. Frente al término «agrícola», la valoración de la economía doméstica como economía agraria subraya la constante vinculación entre ambos elementos. Las especies domésticas aportan una serie de funciones básicas para los campesinos, entre las que destacan el apoyo como fuerza de tiro en labores agrícolas, la reconstitución de la fertili- 
dad de la tierra mediante el abonado, el aporte proteínico a la dieta, el transporte de productos $y$, en su caso, de excedentes.

Partiendo de una contraposición inexistente, la dualidad de hábitat en llano y altura ha sido vista como una dualidad económica de poblaciones diferentes. Se trata de un recurso reiteradamente utilizado por las corrientes normativistas, aunque en el caso madrileño sorprende la inversión de actividades supuestas por algunos investigadores (p.e. Blasco, 1992): pastores en el llano, con el complemento de la caza y en último término de la agricultura; pastores en altura, aunque con intereses económicos como el comercio o la agricultura. La aceptación de esta interpretación proviene, sin lugar a duda, de la conversión de una hipótesis en dogma (Harding, 1980). Todos los datos seleccionados tradicionalmente resultaban fácilmente asimilables desde una hipótesis ganadera. Las incongruentes dificultades derivadas de incluir los yacimientos en altura dentro de dicha hipótesis lleva a concederles diferente función económica e, inevitablemente, diferente grupo poblacional. Los fundamentos de la interpretación son claramente normativistas, en cuanto obvian problemas como la dificultad de aislar elementos diferenciadores entre los yacimientos, al margen de su altitud.

- El uso acrítico de fenómenos históricamente determinados a la hora de interpretar sociedades prehistóricas. La trashumancia continua siendo uno de los recursos argumentales más utilizados por los prehistoriadores peninsulares. Se obvia, sin embargo, que dicho fenómeno representa una adaptación táctica de época medieval, más que una estrategia a largo plazo que deba buscarse en la Prehistoria europea (Lewthwaite, 1981). La trashumancia se asocia al movimiento de grandes rebaños a lo largo de largas distancias, implicando «una división del trabajo, una agricultura omnipresente y, en consecuencia, una continuidad de cultivos, moradas fijas y pueblos» (Braudel, 1976: 112).

La escasamente explícita interpretación defendida hasta la actualidad permite el uso de toda una serie de términos ambiguos, utilizando supuestos sinónimos como nomadismo, trasterminancia, trashumancia, temporalidad o itinerancia, junto con conceptos como «economías mixtas» o «agrícolas marginales». En ningún caso queda determinado el peso específico concedido al factor agrícola dentro del conjunto, mientras se continua defendiendo la determinante importancia del componente animal. Esta ambigüedad permite que todo nuevo dato arqueológico sea absorbido dentro del sistema. Al no existir formulación contrastable, la hipótesis ganadera se presenta como un clásico ejemplo de razonamiento normativo, una de cuyas objeciones continua siendo la imposibilidad de refutar las hipótesis mediante el registro, siendo por tanto científicamente inoperantes.

Frente a ello, el Procesualismo estableció la importancia de un marco teórico que permitiera contrastar las hipótesis generadas con el creciente registro arqueológico. Una de las más sugerentes propuestas, en torno a la evolución socioeconómica en parte de la España seca, ha sido la desarrollada a lo largo de la última década por R.J. Harrison.

\section{UNA TENTATIVA PROCESUAL: EL MODO PASTORIL INTEGRADO DE R. J. HARRISON}

A R.J. Harrison (1985) se debe el primer modelo teórico, en el que se defiende la importancia del componente animal doméstico dentro de las sociedades del III y II milenios a.C. en el Norte y centro de la Península Ibérica. Este modelo de gestión económica, denominado «Policultivo ganadero", se define como «un proceso sistemático de intensificación agrícola en donde los animales son progresivamente utilizados más por su fuerza de tracción, movilidad, estiercol, lana o productos lácteos, que por su carne» (Harrison, 1994: 273). El proceso no se presenta como una auténtica revolución, sino como un efecto acumulativo con dos tendencias previsibles: la concentración de riqueza en menos manos y la regionalización de los patrones económicos basados en el intercambio de animales o de sus productos (Harrison, 1985: 100).

La propuesta pretende explicar uno de los fenómenos más relevantes de la Prehistoria reciente de la Península Ibérica: el desarrollo diferencial (Fig. 1). A pesar de la diversidad de enfoques, existe un amplio consenso en torno a la existencia de formas más o menos consolidadas de desigualdad social en el Suroeste, el Sureste o La Mancha (Gilman, 1981; Lull, 1983; Chapman, 1991; Díaz-Andreu, 1995). Al contrario, el resto de la denominada España seca cuenta con escasos indicadores arqueológicos que 
permitan considerar la existencia de las mismas. Al establecer el centro de su investigación en esta última, Harrison introduce un problema particular dentro de un marco general de debate: los mecanismos generados por las «sociedades primitivas" para impedir el desarrollo de formas de explotación consolidadas.

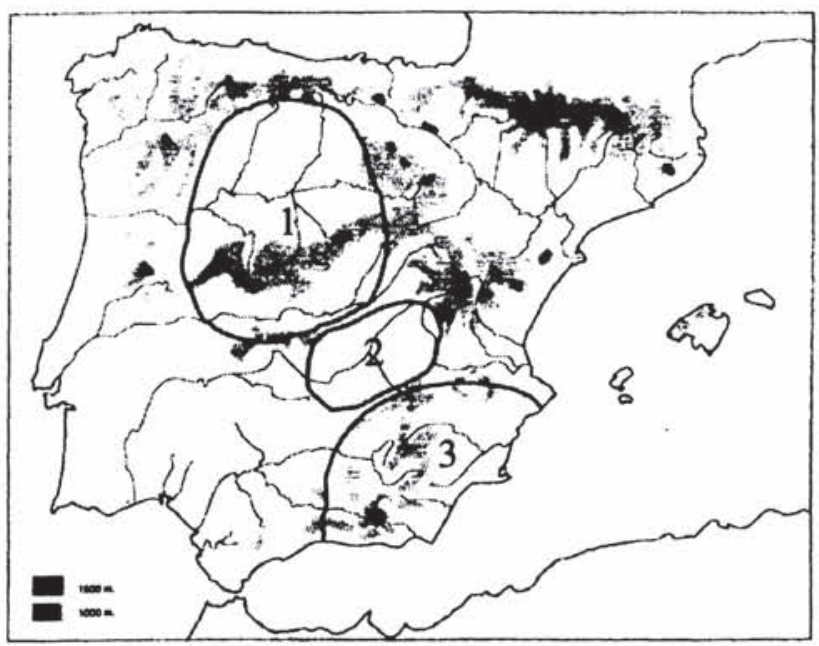

Fig. 1. Desarrollo diferencial en la Península Ibérica durante la Edad del Bronce: 1. Área de análisis. 2. Bronce Manchego. 3. Sudeste.

Según Harrison (1993), los problemas básicos de subsistencia con los que se encuentran este tipo de sociedades parten de factores como la extensión de la tierra y mano de obra disponibles. En este sentido, la mano de obra es considerada el factor clave por cuanto la presión sobre la tierra resulta empíricamente indefendible en gran parte de la España seca durante el III y II milenios a.C. Frente a dicho problema, y siguiendo a Halstead (1989), propone cuatro estrategias que permiten mitigar la carencia de mano de obra: almacenaje, intercambio, diversidad y movilidad, siendo las dos últimas las adoptadas durante la Edad del Bronce. A partir de ello, establece tres momentos de evolución económica dentro de la Prehistoria reciente en la España seca:

- La base económica agrícola combinada, con un importante papel concedido al cerdo, durante el Eneolítico. En este período se desarrollaron amplias redes de intercambio de bienes de prestigio.
- La base agrícola de la Edad del Bronce, con un componente ganadero variado y móvil. Esta propuesta se fundamenta en la reducción de la cabaña porcina y el aumento del caballo, al cual se le concede una repentina importancia, desapareciendo a su vez las redes de intercambio.

- La renovación de las redes de intercambio de bienes de prestigio a partir del 1200 a.C.

La argumentación propone explicar la ausencia de evolución hacia formas permanentes de desigualdad social en la España seca, considerando que la clave de su inexistencia se encuentra en la introducción del elemento pastoril dentro de la economía agrícola. Esta hipótesis se basa en la identificación de transformaciones en el paisaje a partir de datos polínicos obtenidos en el Suroeste peninsular (Stevenson y Harrison, 1992), interpretadas como la implantación, ya desde el Eneolítico, de un sistema de dehesas (2), así como en el análisis de una serie de muestras faunísticas peninsulares (Harrison, 1985).

Las dos premisas de las que parte son: a) que la dehesa es producto de la transformación social del paisaje natural, presentándose como un indicador de la intensificación económica y b) que el elemento pastoril se encuentra plenamente integrado dentro de una economía agrícola. Esta estricta primacía del factor agrícola sobre el ganadero ha sido recientemente matizada por el autor, el cual, estableciendo un paralelismo con las abundantes poblaciones pastoriles conocidas por la etnografía en las cuales la coacción se evita mediante la posesión de bienes móviles, considera que el «elemento ganadero engendra cierta independencia muy codiciada" (Harrison, 1993: 298).

Sin embargo, la propuesta de Harrison resulta matizable si se tienen en cuenta las reflexiones respecto a la clasificación de las sociedades pastoriles y agrícolas realizada por Ingold (1984; 1987). Según dicho autor, el factor trascendental. es determinar si las relaciones sociales dominantes se fundamentan sobre la posesión de medios de producción inmóviles o móviles (básicamente tierra o ganado).

(2) El término "dehesa" (del latín defensa), suele referirse a espacios acotados generalmente de pastos, protegidos frente al avance de las tierras de cultivo. Su carga histórica hace que quizás resulte poco apropiado para la descripción de un paisaje prehistórico. 
Al establecer la primordial importancia del modo pastoril durante la Edad del Bronce. Harrison plantea implícitamente la existencia de un cambio en las relaciones sociales de producción, las cuales pasarían de quedar determinadas por la posesión de la tierra como medio de producción principal a determinarse por la posesión de medios de producción móviles, como mecanismo mitigador del desarrollo de formas permanentes de desigualdad social. Esta supuesta transformación de las relaciones de producción resulta contradictoria con la inversión diferida de fuerza de trabajo necesaria para la creación. conservación y explotación de un sistema de dehesas, la cual ineludiblemente vincularía las relaciones de producción a la posesión o control de inmóviles, es decir, a la tierra. En este sentido, la gestión ganadera de una dehesa como «elemento integrado" depende estrictamente del factor inmóvil, siendo el papel social de la ganadería no tanto determinante como subordinado.

La alternativa propuesta a continuación se diferencia del modelo de integración pastoril por su énfasis en la vinculación de los productores con sus medios de producción inmóviles. Asimismo considera que, en gran medida, el proceso histórico desarrollado en la Meseta peninsular durante la Prehistoria reciente puede explicarse desde el denominado principio de pluriactividad campesina.

\section{LA FORMULACIÓN DEL CONCEPTO DE CAMPESINADO}

La revitalización del análisis de las economías campesinas, a raíz de la reedición de las obras de Chayanov en los años 1960 (Durrenberger, 1984), perfiló un lugar de debate común para investigadores provenientes de las más diversas áreas de conocimiento: antropología, etnología, historia, geografía, sociología, economía o política.

El acceso a puestos académicos de nuevas generaciones de prehistoriadores en la Península Ibérica revitalizó en gran medida el debate en torno a la necesidad de establecer los fundamentos teóricos de la disciplina. Aunque aún queda un amplio espectro de prehistoriadores normativistas, muchos de ellos bajo apariencias funcionalistas (Gilman, 1995), el desarrollo de perspectivas postprocesuales ha sido una de las principales características de la reciente investigación. Sin embargo, la potencialidad de las herramientas analíticas de los Estudios Campesinos han recibido, salvo excepciones (Vicent, 1991a), escasa atención entre los investigadores.

La presente perspectiva pretende sugerir una formulación teórica del concepto de campesino válida para el estudio de las formaciones sociales de la Prehistoria reciente para, a través de ella, proponer una alternativa a las discutidas hipótesis sobre la economía pastoril de la Meseta peninsular durante el III y II milenio a.C.

Analizada desde una perspectiva histórica, la acumulación originaria de capital, por la que el productor se vio desposeído de sus medios de producción (Marx, 1978a), debió ser precedida por una acumulación primitiva, en la cual aquel quedó ineludiblemente vinculado a los mismos (Vicent, 1995). Este fenómeno, conocido como "revolución neolítica», debió culminar en la formación del primer "modo de vida campesino» (Vicent, 1991b; Hurtado, 1995), siendo la consolidación del primer campesinado la principal característica histórica del Calcolítico y la Edad del Bronce.

El campesino es productor directo, poseedor de sus propios medios de producción de los que el principal es la tierra. Caracteriza su modo de vida la ineludible e indisoluble vinculación y dependencia entre el productor y la tierra, su laboratorio natural (Marx, 1985), por oposición a aquellas formaciones sociales en las cuales la tierra representa exclusivamente un objeto de trabajo. Establecer las condiciones necesarias para la transformación de los diversos ecosistemas en medios naturales modificados (Toledo, 1993) implicó no solo un profundo conocimiento de la naturaleza sino una auténtica revolución social. En este proceso, la inversión de trabajo social en el medio ambiente natural transformó al mismo en medio de producción, de tal forma que desde entonces resultase superior el coste de su abandono y de una nueva inversión al mantenimiento de una productividad mínima. En definitiva, el desarrollo de una serie de medios de producción de rendimiento diferido provocó la progresiva dependencia de sus productores. Desde esta perspectiva, la trampa agrícola resultó ser una auténtica trampa social (Vicent, 1991b).

Tradicionalmente los Estudios Campesinos han utilizado la presencia de grupos dominantes que extraen el excedente (p.e. Wolf, 1982: 12) como frontera de demarcación entre las denominadas economías primitivas (Sahlins, 1977) o aldeas agrícolas tribales (Toledo, 1993), y la

\section{T. P., 52, n. ${ }^{\circ} 2,1995$}


"auténtica» comunidad campesina. Sin embargo, esta demarcación resulta matizable desde el punto de vista aquí expuesto. La característica que define la comunidad campesina no es la presencia o ausencia del grupo dominante, sino la existencia de una condición específica que permite el desarrollo del mismo: las ataduras generadas por una inversión de trabajo para una producción diferida.

La racionalidad económica campesina gira en torno a la resolución de su principal problema: la incertidumbre proveniente de una naturaleza impredecible (3). El safety-first peasant (Ellis, 1988) minimiza dicha incertidumbre mediante el principio de la diversidad, que afecta tanto a la producción y reproducción agraria como a la diversificación de la actividad: la pluriactividad campesina (Domínguez Martín, 1992). La adopción de esta estrategia multiuso nada tiene que ver con el campesino maximizador de la producción propuesto por las corrientes neoclásicas (Ellis, 1988: 63-79). Frente a ellas, el denominado Neopopulismo marxista (Sevilla, 1990; Sevilla y González de Molina, 1993) defiende la existencia de un intercambio ecológico, no económico, fundamentado en el desarrollo de una producción no especializada. Este tipo de producción implica el uso de diversos ecosistemas, así como «la integración y combinación de diferentes prácticas, el reciclaje de materias, energía, agua y residuos, y la diversificación de los productos obtenidos" de los mismos (Toledo, 1993: 208). Sin embargo, el campesino no «utiliza» dichos ecosistemas, los manipula de tal forma que consigue mantener una amplia heterogeneidad espacial así como una considerable diversidad biológica (Toledo, 1993: 209).

La seguridad, como hilo racionalizador del comportamiento económico campesino, plantea una primera contradicción en el modo de vida: el necesario equilibrio entre reciprocidad extragrupal negativa y positiva. A pesar del alto componente autosubsistencial de la economía doméstica, la prístina comuna campesina jamás ha respondido a un microcosmos localizado (Marx en Shanin, 1990: 145), resultando necesaria la asociación supradoméstica para la producción y reproducción social.

(3) La utopía campesina de Chayanov, publicada bajo el seudónimo de Ivan Kremnev (1976), necesitó de un artilugio fantástico, el "meteoforo", como solución a la impredecible fluctuación climática.
La reciprocidad negativa conlleva la restricción del acceso a la tierra a todo aquel que no pertenezca al grupo. En estas condiciones, el parentesco genealógico institucionaliza la reciprocidad negativa extragrupal, negando el acceso a la tierra, como conjunto de ecosistemas y medio de producción principal, a todos aquellos que no estén unidos mediante lazos de consanguinidad, impidiendo a su vez la posible «fuga» de fuerza de trabajo. La identidad colectiva del grupo ya no se fundamenta en la referencia a un antepasado mítico, sino a un antepasado genealógico, estableciendo un nuevo mecanismo que asegura la continuidad y reconocibilidad del grupo (Habermas, 1981). Este fenómeno permite hablar de la noción campesina de territorio, entendido, siguiendo a Foucault (1991: 116), como «lo que es controlado por un cierto tipo de poder». Bajo estas condiciones, la asignación libre de los recursos del grupo pasa por un control político del territorio, siendo el ideal autárquico el medio para la afirmación del ideal de independencia política (Clastres, 1987: 196). Es por ello que, en estos términos, puede hablarse de territorio político campesino.

La propia reproducción del grupo convierte en necesaria la defensa del plusproducto. Por cuanto el mantenimiento de un sistema agrario exitoso supone la previsión de un fondo de seguridad y reproducción, el almacenaje se convierte en un factor indispensable en la economía doméstica campesina. En este caso, la función de la reciprocidad negativa es impedir el acceso al plusproducto a todo componente foráneo y aplazar el acceso del grupo productivo al mismo.

Frente a ello, la reciprocidad positiva exige el establecimiento de un sistema capilar de relaciones políticas intercomunitarias. La seguridad campesina considera como factores imprescindibles para su subsistencia tanto la reproducción del grupo doméstico como el potencial apoyo externo en caso de una pérdida máxima en la producción doméstica. La trascendencia de la reproducción se encuentra en el reparto de descendencia como futura fuerza de trabajo, desarrollando lo que ha venido en denominarse un «intercambio igual» de reciprocidad absoluta (Meillassoux, 1987). A su vez, el peligro de una crisis subsistencial lleva al campesino a rechazar el riesgo, minimizando la probabilidad de una crisis subsistencial antes de maximizar su rendimiento medio (Domínguez Martín, 1992). Este último fenómeno refiere directamente a una 
característica del campesinado originalmente propuesto por Chayanov: su rechazo a lo que es percibido como un esfuerzo innecesario. Mientras no exista la necesidad de recurrir a otro grupo, la unidad doméstica se ve en la tesitura de conservar los vínculos intercomunitarios, siendo la forma más frecuente de acción la visita a los vecinos, el intercambio de bienes de uso o la organización de festines con la finalidad de agasajarlos.

Las características expuestas han eludido, sin embargo, un factor fundamental dentro del proceso histórico. La vinculación campesino-tierra trasciende la mera clasificación, por cuanto la "trampa agrícola" consumada (Vicent, 1991b: 45) establece las bases que permiten el desarrollo de formas de coacción extraeconómica (Marx, 1978b). Al resultar excesivamente costoso el abandono de un medio de producción en el que se ha invertido amplias cantidades de trabajo social, el campesino se convierte en presa fácil para el poder externo (Meillassoux, 1973). Esta perspectiva permite explicar el desarrollo de episodios de la Prehistoria reciente en los cuales la movilización de fuerza de trabajo hacia factores no productivos (murallas, fortines...) crea espacios de coerción arqueológicamente registrables. La imposibilidad de perpetuar a largo plazo el poder en una sociedad que sólo admite el prestigio (Clastres, 1987), la incapacidad para acumular valor, así como la no descartable resistencia campesina (4) (Scott, 1986; Gilman, 1987) lleva al fracaso de estas "culturas». Sin embargo, el campesinado, sobre el cual recaen todos estos sistemas prototributarios, se conserva como personaje continuo del proceso histórico.

\section{LA ESTRATEGIA AGROFORESTAL: UN MODELO DE EXPLOTACIÓN ECONÓMICA DURANTE LA PREHIS- TORIA RECIENTE DE LA MESETA PENINSULAR}

Las condiciones específicas de la Meseta peninsular han permitido el desarrollo histórico

(4) Entendemos la resistencia campesina no como un movimiento organizado con expresión política (Hobsbawn, 1973), sino como formas no coordinadas, siquiera necesariamente colectivas, de resistencia diaria (Scott, 1986): ratería, furtivismo, ignorançia, etc. Esta conceptualización de la resistencia tiene su antecedente más directo en la "economía moral de la multitud" desarrollada por E.P. Thompson (1979). de un sistema de gestión campesina del ecosistema: la estrategia agroforestal. Se entiende como tal el «uso de la tierra en donde las plantas leñosas (árboles y matorrales) crecen deliberadamente en la misma unidad de suelo con cultivos agrícolas y/o animales, y donde existen, simultáneamente interacciones ecológicas y económicas entre los diferentes componentes" (Stocking en Campos Palacín, 1994). La estrategia permite el acceso tanto a recursos domésticos como silvestres, sean animales o vegetales, combinación que concede a la comunidad campesina la requerida seguridad, intrínseca a una concepción de la producción en la cual se minimiza el riesgo.

Este tipo de gestión del territorio implica un conocimiento tan profundo de la diversidad del ecosistema como para invertir la necesaria fuerza de trabajo social en su mantenimiento. Un sistema agroforestal no puede ser entendido como el uso racional de un medio ambiente natural, sino como la combinación y el equilibrio racional entre un medio ambiente natural y modificado. Por ello, las comunidades campesinas con gestión agroforestal, agregados conscientes de grupos domésticos interrelacionados (Sevilla y González, 1993), pueden llegar a encontrarse tan vinculadas a su territorio como lo estan aquellas formaciones sociales campesinas en las cuales la gestión se realiza fundamentalmente mediante la inversión de trabajo social en sistemas de regadío.

La gestión agroforestal requiere la manipulación combinada del ecosistema, del cual se extrae una amplia variedad de productos: alimentos silvestres y agrícolas, herramientas de producción, instrumentos domésticos, alimentos para los animales, combustibles, así como gomas, resinas, colorantes, medicamentos y estimulantes (Toledo, 1993: 209). Desde esta perspectiva puede entenderse que las estrategias de diversificación, almacenaje, intercambio o movilidad (Halstead, 1989) sean asumidas en su conjunto, y no necesariamente de forma individual, por cuanto todas ellas son componentes de este modo de vida:

- La diversificación indica el conocimiento y manipulación de vegetales silvestres, la producción y reproducción del ciclo agrícola, el control social de la reproducción de especies domésticas y el acceso a recursos cinegéticos presentes dentro del territorio político campesino.

- El almacenaje de restos vegetales silvestres y agrícolas se convierte en un factor funda- 
mental en la economía campesina. Es un fondo de seguridad establecido mediante un mecanismo social de acceso diferido a la producción, que permite a su vez la renovación del ciclo ecológico modificado.

- El intercambio se presenta como materialización de la reciprocidad positiva intergrupal, sin que necesariamente circulen herramientas de producción o elementos básicos dentro del proceso productivo. Este tipo de intercambios no deben ser entendidos como flujos unidireccionales, sino como una red dendriforme de carácter multidireccional, en la que se encuentra comprometida la totalidad de las unidades domésticas.

- La movilidad a corto, medio o largo plazo, factor recurrente en las «hipótesis ganaderas", puede ser tanto indicativa de la pluriactividad campesina como un elemento común a gran parte de las sociedades precapitalistas. El acceso a diversos tipos de productos en diversos momentos del año puede crear un patrón de distribución de yacimientos similar al documentado en gran parte de la Meseta peninsular. Esta movilidad no tiene por qué referirse a la totalidad del grupo doméstico, por cuanto los factores inmóviles básicos tenderán a situarse en las proximidades del hábitat agrario, un ejemplo más de la minimización de esfuerzo característica de las sociedades campesinas.

Como proceso histórico, el origen del uso de sistemas agroforestales de gestión del territorio puede paralelizarse con lo definido como Revolución de los Productos Secundarios (Sherrat, 1981) en su forma meseteña. Como afirma Harrison (1993: 294), la intensificación es «una realidad más vinculada al ejercicio de una opción, para manipular el orden social a largo plazo, que a los cambios económicos». Sin embargo, esta manipulación del orden social no debe ser asumida desde un evolucionismo lineal, por el que se llegaría ineludiblemente al establecimiento de una sociedad de clases. La estrategia multiuso se encuentra específicamente vinculada al concepto de seguridad campesina, y como tal mecanismo, pretende manipular el orden social con una finalidad inmovilista. El campesino no es en exclusiva ecológicamente conservador (González y Sevilla, 1993: 96) sino también, y sobre todo, socialmente conservador. Esta condición ha llevado a que gran parte de los analistas comprometidos con la transformación social revolucionaria hayan, de diversas maneras, vinculado al campesinado con una forma reaccionaria de producción económica (5). En todo caso la intensificación no manipula de por si el orden social, sino que puede establecer las bases para el desarrollo de formas permanentes de desigualdad social, entendidas como la presencia de relaciones de explotación consolidadas, fundamentadas en la coerción extraeconómica.

\section{CÓNCLUSIÓN}

El desarrollo de la investigación prehistórica en las últimas décadas, volcada en interpretar los procesos sociales del Sudeste, ha marcado en gran medida los modelos de explicación histórica aplicados al resto de la Península Ibérica. Sin embargo, la presencia de los indicadores arqueológicos tradicionalmente utilizados para identificar la desigualdad social resultan escasos en la Meseta peninsular, siendo actualmente problemático defender la existencia de una apropiación diferencial permanente, tanto de plusproducto como de plustrabajo.

La resolución de este problema histórico pasa por dos alternativas no excluyentes: la búsqueda de otro tipo de indicadores arqueológicos o la aceptación de una hipotética evolución autónoma del campesinado en la Meseta. Esta última propuesta incidiría en la existencia, en estos grupos sociales campesinos, de los suficientes mecanismos como para mitigar el desarrollo de formas de explotación consolidadas, abriendo a su vez un camino no investigado hasta la actualidad: la gestión agroforestal como una forma originaria de resistencia campesina frente al poder.

\section{AGRADECIMIENTOS}

El trabajo se debe en gran medida a la conti- . nua orientación recibida del Dr. Juan Manuel Vicent (C.E.H., C.S.I.C.) en la cuestión de los denominados «Estudios Campesinos», asi como

(5) Nos referimos al intenso debate desarrollado inicialmente por Marx y los Populistas rusos (Shanin, 1990), el posterior debate sobre la Cuestión Agraria en la que participaron figuras como V.I. Lenin (1981) o K. Kautski (1974), o las más recientes formulaciones del potencial revolucionario campesino desarrolladas por Mao y los diversos seguidores de su doctrina política (p.e. Bettelheim, 1978). 
de la Dra. M" Isabel Martínez Navarrete (C.E.H.. C.S.I.C.), que supo limar con extrema paciencia muchos de mis achaques empiristas. Asimismo, agradezco la lectura crítica que de una primera versión del texto realizaron la Dra. Isabel Rubio, el Dr. Arturo Morales y la Dra. Corina Liesau (U.A.M.). Aunque hubiera deseado compartirla, la autoría final es mía.

\section{BIBLIOGRAFÍA}

Almagro-Gorbea. M. y Fernandez Gallano, D. (1980): "Excavaciones en el Cerro del Ecce Homo (Alcalá de Henares, Madrid)". Arqueología 2. Diputación Provincial de Madrid. Madrid.

Almagro-Gorbea, M. y Benito-López. J.E. (1993): «La prospección arqueológica del Valle del Tajuña. Una experiencia teórico-práctica de estudio territorial en la Meseta». Complutum, 4: 297-310.

ANTONA, V. (1987): "El Neolítico". En "1.30 Años de Arqueologia Madrileña». Comunidad de Madrid. Madrid: 45-57.

BETTELheim. C. (1978): "Las luchas de clases en la URSS". Versión Española de F. Claudín. II vols. Siglo XXI. Madrid.

BlasCO. Mª.C. (1992): “Etnogénesis de la Meseta Sur». Complutum, 2-3: 281-297.

- (1993): «El Bronce Final». Historia Universal, 7. Editorial Síntesis. Madrid.

BLASCO, Ma.C. (ed.) (1994): El horizonte campaniforme de la región de Madrid en el centenario de Ciempozuelos. Patrimonio Arqueológico del Bajo Manzanares, 2. Universidad Autónoma de Madrid. Madrid.

Blasco, Ma C.; Calle. J. y SÁnchez-Capilla. Ma ${ }^{a}$. (1991): "Yacimiento del Bronce Final y de época romana en Perales del Río (Getafe, Madrid)». Arqueología, Paleontología y Etnografía, 1: 37-147. Comunidad de Madrid. Madrid.

BRAUDEL, F. (1976): «El Mediterráneo y el mundo mediterráneo en la época de Felipe II». Fondo de Cultura Económica. Madrid. 2 vols.

Campos Palacin, P. (1994): «El valor económico total de los sistemas agroforestales». Agricultura y Sociedad, 71 (Abril-Junio): 243-256. Madrid.

ChapMAN, R.W. (1979): "Transhumance and megalithic tombs in Iberia». Antiquity, 53: 150-151.

- (1991): "La formación de las sociedades complejas». Crítica. Barcelona.

Clastres. P. (1987): «Investigaciones en Antropología Política». Gedisa. México.

Diaz-Andreu, M. (1995): «Late Prehistoric Social Change in the Southern Meseta of the Iberian Peninsula». En K.T. Lillios (ed.): «The Origins of Complex Societies in Late prehistoric Iberia». International Monographs in Prehistory. Archaeological Series, 8. Ann Arbor. Michigan: 97-120.

Dominguez, R.Ma'; Fernández, A.; Herce, J.L.; MenaSANCH, M. y PRESAS, M. (1993): «Empresas de Arqueo- logía y Arqueología urbana: investigación, negocio. profesión". Arqueología y Territorio Medieval, 1: 8.3-91. Jain.

Domingliez MARtin. R. (1992): “Campesinos, mercado y adaptación. Una propuesta de síntesis e interpretación desde una perspectiva interdisciplinar". Noticiario de Historia Agraria. 3: 91-130. Murcia.

DURRENBERGER. E.P. (ed.) (1984): “Chayanov, peasants and Economic Anthropology". Academic Press. Oriando.

EI.LIS. F. (1988): "Peasant Economics. Farm households and agrarian development". Cambridge University Press. Cambridge.

Foucault, M. (1991): “Microfísica del Poder». Genealogía del Poder. Ediciones de La Piqueta. Madrid.

Gilman. A. (1981): "The development of social stratification in Bronze Age Europe». Current Anthropology, 22 (1): 1-23.

- (1987): "Regadío y conflicto en sociedades acéfalas". Boletín del Seminario de Estudios de Arte y Arqueologia. LIII: 59-72. Valladolid.

- (1995): "Recent Trends in the Archaeology of Spain». En K.T. Lillios (ed.): «The Origins of Complex Societies in Late prehistoric Iberia». International Monographs in Prehistory. Archaeological Series, 8. Ann Arbor. Michigan: 1-6.

Gonzalez de Molina, M. y Sevilla Guzmán, E. (1993): «Ecología, campesinado e historia: Para una reinterpretación del desarrollo del capitalismo en la agricultura». En E. Sevilla Guzmán y M. Gonzalez de Molina (eds.): «Ecología, Campesinado e Historia». Genealogía del Poder, 22. Ediciones de La Piqueta. Madrid: 23129.

HABERMAS, J. (1981): «La reconstrucción del materialismo histórico». Taurus. Madrid.

HALSTEAD, P. (1989): «The economy has a normal surplus: economic stability and social change among early farming communities of Thessaly, Greece». En P. Halstead y J. O'Shea (eds.): «Bad Year Economics». Cambridge University Press. Cambridge: $68-80$.

HARDING. A.F (1980): "Radiocarbon calibration and the chronology of the European Bronze Age». Archeologické Rozhledy, 32. Praga: 178-185.

HARRISON, R.J. (1985): «The 'Policultivo Ganadero', or the Secondary Products Revolution in Spanish Agriculture, 5000-1000 bc». Proceedings of the Prehistoric Society, 51: 75-102.

- (1993): «La intensificación económica y la integración del modo pastoril durante la Edad del Bronce». $1^{\circ}$ Congresso de Arqueologia Peninsular (Oporto, 1993). II. Trabalhos de Antropologia e Etnologia, 33 (3-4): 293299. Oporto.

- (1994): «New aspects of the 'Policultivo Ganadero' in Prehistoric Spain». Origens, Estruturas e Relações das Culturas Calcoliticas da Península Ibérica (Torres Vedras, 1987). Trabalhos de Arqueología, 7. IPPAR. Lisboa: 273-276.

Hobsbawn, E. (1973): «Peasants and Politics». Journal of Peasant Studies, 1: 3-22. 
HURTADO, V. (ed.) (1995): "El Calcolitico a dehate. Re'unión de Calcolitico de la Peninsula Ibérica" (Sevilla. 199()). Junta de Andalucia. Sevilla.

INGOLD. T. (1984): «Time, social relationships and the exploitation of animals: Anthropological reflections on Prehistory». En J. Clutton-Brock y C. Grigson (eds.): Animals and Archaeology: Early herders and their flocks. British Archaeological Reports, International Series, 202. Oxford: 3-12.

- (1987): "Changing places: movement and locality in hunter-gatherer and pastoral societies". En T. Ingold (ed.): "The appropiation of nature. Essays on human ecology and social relations". University of Iowa Press. lowa: 165-197.

KAUTSKI, K. (1974): «La cuestión agraria». Editorial Laia. Barcelona.

KREMNEV, I. (1976): "The journey of my brother Alexei to the land of Peasant Utopia". Journal of Peasant Studies, 4 (1): 63-108.

LENIN, V.I. (1981): "El desarrollo del Capitalismo en Rusia». Obras Completas, III. Editorial Progreso. Moscú.

LEWTHWAITE, J. (1981): «Plain tails from the hills: Trashumance in Mediterranean Archaeology". En A. Sheridan y G. Bailey (eds.): Economic Archaeology. British Archaeological Reports, International Series, 96. Oxford: 57-66.

Lull, V. (1983): "La 'Cultura' de El Argar. Un modelo para el estudio de las formaciones enonómicosociales Prehistóricas". Akal. Madrid.

Martínez NavarRete. M.I. (1984): «El comienzo de la metalurgia en la provincia de Madrid: La cueva y cerro de Juan Barbero (Tielmes, Madrid)». Trabajos de Prehistoria, 41: 17-128. Madrid.

- (1985): «La Edad del Bronce en la Submeseta suroriental: una revisión crítica». Serie Tesis Doctorales. Universidad Complutense de Madrid.

- (1989): «Una revisión crítica de la prehistoria española: la Edad del Bronce como paradigma». Siglo XXI. Madrid.

MARX, K. (1978)a: «El Capital. Crítica de la Economía Política. Libro I: El proceso de producción de capital». $2^{\mathrm{a}}$ edición, $13^{\mathrm{a}}$ reimpresión. Versión castellana de $\mathrm{W}$. Roces. Fondo de Cultura Económica. México.

- (1978)b: "El Capital. Crítica de la Economía Política. Libro III: El proceso global de la producción capitalista». $2^{\mathrm{a}}$ edición, $13^{\mathrm{a}}$ reimpresión. Versión castellana de W. Roces. Fondo de Cultura Económica. México.

- (1985): "Grundrisse. Lineamientos fundamentales para la crítica de la economía política. 1857-1858». I. Versión castellana de W. Roces. Fondo de Cultura Económica. México.

Meillassoux, C. (1973): "The social organisation of the peasantry: the economic basis of kinship". Journal of Peasant Studies, 1 (1): 81-90.

- (1987): «Mujeres, graneros y capitales». Siglo XXI. Madrid.
PERNiA, A. y LFIRA, R. (1992): „Excavaciones de urgencia en el Arenero del Soto II (P.K. $5+360$ ) al P.K. $5+380$ del Tren de Alta Velocidad Madrid-Sevilla)". Arqueologia, Paleontologia y Etnografia. 3. Comunidad de Madrid. Madrid: 117-130.

Priego, C. y Quero, S. (1992): «El Ventorro, un poblado prehistórico de los albores de la metalúrgian. Estudios de Prehistoria y Arqueología Madrileñas, 8. Madrid.

RUS. I. y VELASCO. F. (1993): “El poblamiento prehistórico en Madrid». En A. Fernández García (dir.): "Historia de Madrid». Editorial Complutense. Madrid: 67-86.

Sahlins. M. (1977): "Economía de la Edad de Piedra". Akal Editor. Madrid.

ScOTT. J.C. (1986): "Everyday forms of Peasant Resistance». Journal of Peasant Studies, 2: 5-35.

Sevill.a Guzmán. E. (1990): «Redescubriendo a Chayanov: hacia un neopopulismo ecológico". Agricultura y Sociedad, 55 (Abril-Junio): 201-237.

Sevilla Guzmán, E. y González de Molina, M. (1993): "Ecología, Campesinado e Historia». Genealogía del Poder, 22. Ediciones de La Piqueta. Madrid.

ShANIN, T. (1990): «El Marx tardío y la vía rusa». Editorial Revolución. Madrid.

SHERRATT, A. (1981): «Plough and pastoralism: aspects of the secondary products revolution». En I. Hodder, G. Isaac y N. Hammond (eds.): "Patterns of the Past. Studies in honour of David Clarke». Cambridge University Press: 261-305.

STEVENSON, A.C. y HARRISON, R.J. (1992): «Ancient forest in Spain: a model for land-use and dry forest management in south-west Spain from 4000 BC to 1900 ADm. Proceedings of the Prehistoric Society, 58: 227-247.

THOMPSON, E.P. (1979): «Tradición, revuelta y consciencia de clase. Estudios sobre la crisis de la sociedad preindustrialm. Crítica. Barcelona.

TOLEDO, V.M. (1993): «La racionalidad ecológica de la producción campesina». En E. Sevilla Guzmán y M. González de Molina (eds.): «Ecología, Campesinado e Historia». Genealogía del Poder. Ediciones de La Piqueta. Madrid: 197-218.

VICENT. J.M. (1991)a: «Fundamentos teórico-metodológicos para un programa de investigación arqueo-geográfica». En P. López (ed): «El Cambio Cultural del IV al II milenios a.C. en la Comarca noroeste de Murcia.». I. CSIC. Madrid: 31-177.

- (1991)b: «El Neolítico, transformaciones sociales y económicas». Boletín de Antropología Americana, 24: 3161.

- (1995): «Early social complexity in Iberia. Some theoretical remarks». En K.T. Lillios (ed.): «The Origins of Complex Societies in Late prehistoric Iberia». International Monographs in Prehistory. Archaeological Series, 8. Ann Arbor. Michigan: 177-185.

Wolf, E. (1971): «Los Campesinos». Editorial Labor. Barcelona. 